\section{Optical imaging of reporter gene expression using a positron-emission- tomography probe}

\author{
Hongguang Liu, ${ }^{\mathrm{a}, \mathrm{b}}$ Gang Ren, ${ }^{\mathrm{a}}$ Shuanglong Liu, ${ }^{\mathrm{a}}$ Xiaofen \\ Zhang, $^{\mathrm{a}, \mathrm{b}}$ Luxi Chen, ${ }^{\mathrm{a}}$ Peizhen Han, ${ }^{\mathrm{b}}$ and Zhen Cheng ${ }^{\mathrm{a}}$ \\ ${ }^{a}$ Stanford University, Molecular Imaging Program, Department of \\ Radiology and Bio-X Program, Stanford, California, 94305 \\ ${ }^{\mathrm{b}}$ Tsinghua University, Peking Union Medical College, Chinese \\ Academy of Medical Sciences, Institute of Radiation Medicine \\ Tianjin,300192, China
}

\begin{abstract}
Reporter gene/reporter probe technology is one of the most important techniques in molecular imaging. Lately, many reporter gene/reporter probe systems have been coupled to different imaging modalities such as positron emission tomography (PET) and optical imaging (OI). It has been recently found that OI techniques could be used to monitor radioactive tracers in vitro and in living subjects. In this study, we further demonstrate that a reporter gene/nuclear reporter probe system [herpes simplex virus type-1 thymidine kinase (HSV1-tk) and 9-(4-18 F-fluoro3 -[hydroxymethyl] butyl) guanine $\left.\left(\left[{ }^{18} \mathrm{~F}\right] \mathrm{FHBG}\right)\right]$ could be successfully imaged by $\mathrm{Ol}$ in vitro and in vivo. OI with radioactive reporter probes will facilitate and broaden the applications of reporter gene/reporter probe techniques in medical research. (O) 2010 Society of Photo-Optical Instrumentation Engineers. [DOI: 10.1117/1.3514659]
\end{abstract}

Keywords: biomedical optics; imaging; technology; radioactive optical imaging; reporter gene; Cerenkov radiation.

Paper 10474LR received Aug. 27, 2010; revised manuscript received Oct. 17, 2010; accepted for publication Oct. 19, 2010; published online Nov. 24, 2010.

Molecular imaging combines molecular biology and medical imaging, allowing the visualization of cellular processes in living subjects at the molecular level. Reporter gene/reporter probe technology is a powerful molecular imaging technique that provides a generalizable method for noninvasive imaging of protein expression, protein function, and protein-protein interaction. ${ }^{1}$ Reporter gene imaging has been widely used in biomedical research to address many fundamental biological problems, including monitoring the progress of cancers, screening drugs, monitoring gene therapy, and tracking the fate of cells. ${ }^{2}$

Many reporter gene/reporter probe systems have been coupled with different imaging modalities over the past two decades. For reporter gene optical bioluminescence imaging (BLI), luciferase as a reporter gene with D-luciferin or coelenterazine as the substrate has been widely used. ${ }^{2}$ Because of the extremely low background, this imaging system can monitor luminescence

Address all correspondence to: Zhen Cheng, Molecular Imaging Program at Stanford, Department of Radiology and Bio-X Program, Stanford University Stanford, CA 94305. Tel: (650) 723-7866; Fax: 650-721-6712; E-mail: zcheng@stanford.edu. light with high sensitivity. However, the light produced from the enzyme/substrate reaction usually has a peak less than $600 \mathrm{~nm}$ and thus displays poor tissue penetration. ${ }^{3}$ For nuclear imaging, a well-established radionuclide-based imaging reporter gene/reporter probe system is the herpes simplex virus type-1 thymidine kinase (HSV1-tk) enzyme, and radio-labeled uracil nucleoside or acycloguanosine derivatives such as $9-\left(4-{ }^{18} \mathrm{~F}\right.$ fluoro-3-[hydroxymethyl] butyl)guanine ([$\left.\left.{ }^{18} \mathrm{~F}\right] \mathrm{FHBG}\right)$, or $2^{\prime}$ $\left[{ }^{18} \mathrm{~F}\right]$ fluoro-5-ethyl-1- $\beta$-D-arabinofuranosyluracil $\left(\left[{ }^{18} \mathrm{~F}\right] \mathrm{FEAU}\right)$ as the reporter probe. ${ }^{4,5}$ Another is the sodium iodide symporter and the reporter probe radioiodine or ${ }^{99 \mathrm{~m}} \mathrm{Tc}$-pertechnetate. ${ }^{6,7}$ In using these approaches, several disadvantages arise, namely that nuclear reporter gene systems require positron emission tomography (PET) or single photon emission computed tomography (SPECT), which are expensive and may not be easily accessible to many researchers.

Recently, we and others have found that a variety of radioactive materials ( $\beta^{+}$and $\beta^{-}$emitters) could be detected by OI techniques. It has been successfully demonstrated that radioactive molecular probes such as 2-deoxy-2-[ $\left[{ }^{18} \mathrm{~F}\right]$ fluoro-D-glucose ([ $\left.\left.{ }^{18} \mathrm{~F}\right] \mathrm{FDG}\right), \mathrm{Na}^{18} \mathrm{~F}, \mathrm{Na}^{131} \mathrm{I},{ }^{90} \mathrm{YCl}_{3}$, and ${ }^{90} \mathrm{Y}$ labeled tumor targeting peptides could be noninvasively imaged in small animals by optical imaging instruments. This is mainly attributed to the ability of radioactive materials to produce low energy visible photons (1.2 to $3.1 \mathrm{eV}, 400$ to $1000 \mathrm{~nm}$ ) associated with Cerenkov or Bremsstrahlung radiation. ${ }^{8-11}$ In this research study, we further evaluated the feasibility of using OI to monitor the nuclear reporter gene/reporter probe systems. HSV1-tk and $\left[{ }^{18} \mathrm{~F}\right] \mathrm{FHBG}$ was thus chosen as a model system in this study. The C6 rat glioma cell line stably transfected with HSV-sr39tk plasmids (C6-tk) (12) was used, and the in-vitro $\left[{ }^{18} \mathrm{~F}\right] \mathrm{FHBG}$ uptakes in C6-tk cells were measured by both an IVIS optical imaging system (Caliper, Hopkinton, Massachusetts) and a gamma counter (Perkin Elmer, Waltham, Massachusetts). Finally, we performed in-vivo imaging studies of the reporter gene/reporter probe using both OI and PET, followed by a biodistribution study.

The in-vitro $\left[{ }^{18} \mathrm{~F}\right] \mathrm{FHBG}$ cell uptake study was performed as previously described with minor modifications. ${ }^{12}$ Briefly, C6-tk cells were seeded into 12-well plates at a density of $5 \times 10^{5}$ cells per well $12 \mathrm{~h}$ prior to the experiment. The C6 cell line without transfection was used as a negative control. The cells were then incubated with $\left.{ }^{18} \mathrm{~F}\right] \mathrm{FHBG}\left(\sim 10^{7} \mathrm{kBq} /\right.$ well, $3 \mu \mathrm{Ci} /$ well $)$ at $37^{\circ} \mathrm{C}$ for $15,30,60$, and $120 \mathrm{~min}$. Tumor cells were washed three times with chilled phosphate buffered saline (PBS) and harvested with $0.25 \%$ trypsin $/ 0.02 \%$ ethylenediaminetetraacetic acid (EDTA) (Invitrogen, Carlsbad, California). The cell suspensions were transferred to a 96-well flat bottom black plate (Nunc) and imaged by an IVIS 200 system (Caliper). Luminescent images were taken without a filter [see Fig. 1(a)]. The exposure time was $5 \mathrm{~min}$. Then, the radioactivity of the cell suspension was measured in a gamma counter (Packard, Meriden, Connecticut). The in-vitro cell uptake results were expressed as the percentage of the applied activity with decay correction [see Fig. 1(b)]. Experiments were performed twice with triplicate samples for each time point. Both OI and gamma counting show a rapid and high uptake of $\left[{ }^{18} \mathrm{~F}\right] \mathrm{FHBG}$ in C6-tk cells, whereas nontransfected C6 cells show very low

1083-3668/2010/15(6)/060505/3/\$25.00 @ 2010 SPIE 
(a)

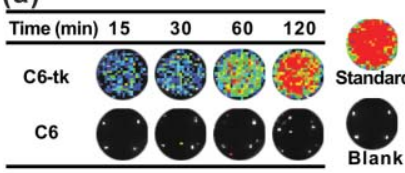

(c)
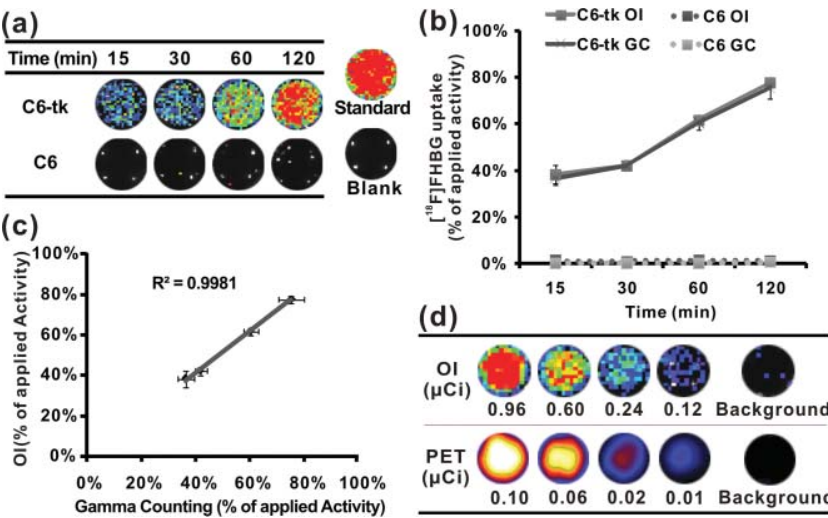

(d)

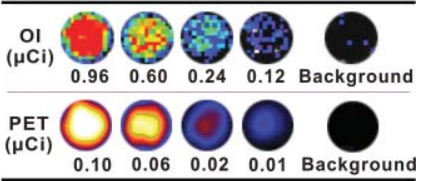

Fig. 1 In-vitro cell imaging (a), (b), and (c) and sensitivity comparison studies of OI and PET (d). Shown are (a) C6 cell uptakes monitored by $\mathrm{OI}$; (b) quantification and (c) correlation of $\mathrm{C} 6$ cell uptakes from both $\mathrm{OI}$ and gamma counting (GC) results; and (d) different radioactivity monitored by OI and PET [OI: 0.96, 0.60, 0.24 and $0.12 \mu \mathrm{Ci}(35.48$, 22.18, 8.88 and $4.44 \mathrm{kBq})$; PET: $0.10,0.06,0.02$ and $0.01 \mu \mathrm{Ci}(3.55$, $2.22,0.89$ and $0.44 \mathrm{kBq})](n=4)$.

cell uptake [see Figs. 1(a) and 1(b)]. More importantly, an excellent correlation has been obtained between OI and gamma counting results [see Fig. 1(c), $r^{2}>0.95$ ]. These findings lay the foundation for further in vivo studies to validate the feasibility of using OI for monitoring the nuclear reporter gene system.

Prior to in-vivo imaging, comparison studies were performed to evaluate the detection sensitivity of the IVIS 200 OI system (Caliper) and the small animal PET (microPET R4 rodent model scanner, Siemens Medical Solutions USA, Washington, D.C.). Serial samples containing different amounts of radioactivity $\left(\mathrm{Na}^{18} \mathrm{~F}\right)$ were prepared in $300 \mu \mathrm{l}$ of water (ranged from 0.01 to $1 \mu \mathrm{Ci}$ ) and imaged by OI and PET [see Fig. 1(d)]. A 5-min imaging time was applied to both modalities. For PET, signal-to-noise ratio (SNR) of 32.1 is obtained from $0.01 \mu \mathrm{Ci}$ $(0.44 \mathrm{kBq})$ of ${ }^{18} \mathrm{~F}$, while OI can detect $0.1 \mu \mathrm{Ci}(4.4 \mathrm{kBq})$ of ${ }^{18} \mathrm{~F}$ with a SNR of 1.7. It was found that ${ }^{18} \mathrm{~F}$ samples lower than $0.1 \mu \mathrm{Ci}$ could hardly be differentiated from background in OI images. PET excels OI in both sensitivity and imaging contrast. The difference in sensitivity between the two modalities could be attributed to the weak photon production of radionuclides and thus low signal intensities from ${ }^{18} \mathrm{~F}$ in OI. Although less sensitive than PET, OI demonstrates promising detection capability and warrants further in-vivo experiments.

All animal studies were carried out in compliance with federal and local institutional rules for the conduct of animal experimentation. Mice bearing two tumors (C6-tk and C6) were then used for in-vivo studies. The C6-tk (left) and C6 (right) cells were injected contralaterally in the dorsal shoulder region of each mouse $\left(2 \times 10^{6}\right.$ cells/mouse; athymic nude mice were purchased from Charles River Laboratories, Wilmington, Massachusetts, $n=6$ ). Approximately 10 days later, when tumor size reached about 1 to $1.5 \mathrm{~cm}$ in diameter, small animal PET and OI were performed using the mice and the PET probe $\left[{ }^{18} \mathrm{~F}\right] \mathrm{FHBG}$. Images at 1 and $2 \mathrm{~h}$ were obtained by both OI and PET. Results are shown by average radiance (photon $/ \mathrm{second} / \mathrm{cm}^{2} / \mathrm{str}$ ) for OI and percentage of injected dose per gram $(\% \mathrm{ID} / \mathrm{g})$ for PET based on the method previously described. ${ }^{13}$ At $1 \mathrm{~h}$ after tail vein injection of $\left[{ }^{18} \mathrm{~F}\right] \mathrm{FHBG}(10$ (a)

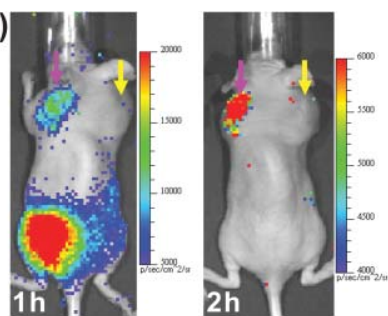

(b)

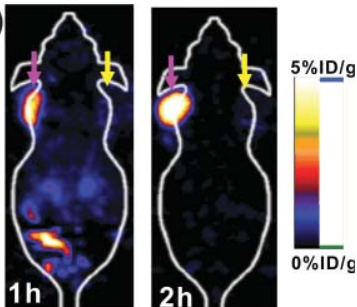

$\downarrow \mathrm{C} 6-\mathrm{tk} \quad \downarrow \mathrm{C} 6$

(c)

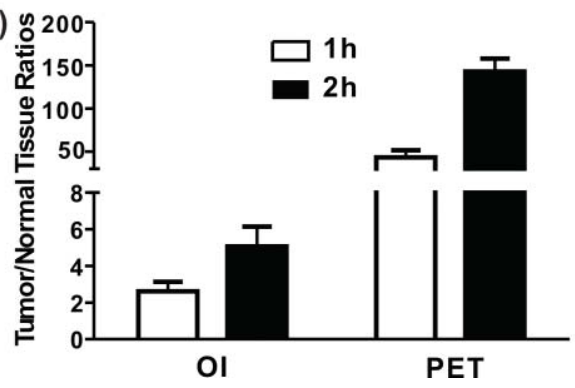

Fig. 2 In-vivo imaging using OI and PET. Mice bearing C6-tk and C6 tumors can be imaged by both (a) OI and (b) PET. (c) Statistical analysis was performed for both modalities $(n=6)$.

to $11 \mathrm{MBq}, 270$ to $300 \mu \mathrm{Ci}$ ), C6-tk tumors can be clearly delineated using OI, while there is minimum tracer uptakes in the control C6 tumors (see Fig. 2). The strong signals in the lower part of the mouse body [Fig. 2(a)] are due to the presence of the radioactivity in the bladder. PET imaging shows a similar pattern of tracer distribution [Fig. 2(b)]. At 2-h postinjection, with the elimination of $\left[{ }^{18} \mathrm{~F}\right] \mathrm{FHBG}$, only C6-tk tumors can be clearly visualized in both imaging modalities (see Fig. 2). Figure 2(c) shows the statistical analysis of tumor to normal tissue (T/N) ratio for both imaging modalities at 1 - and 2-h postinjection. Compared to OI, PET displays higher T/N ratios (OI: $2.6 \pm 0.5$ at $1 \mathrm{~h}, 5.1 \pm 1.1$ at $2 \mathrm{~h}$; PET: $43.2 \pm 8.4$ at $1 \mathrm{~h}, 143.0 \pm 14.8$ at $2 \mathrm{~h}$ ).

To further compare the imaging results obtained from OI and PET, biodistribution studies were conducted. Mice were sacrificed and different organs were collected at 1-h postinjection of $\left[{ }^{18} \mathrm{~F}\right] \mathrm{FHBG}$. OI images were obtained for these radioactive organs, and quantification analysis of optical images was performed using Living Image software version 3.1 [see Fig. 3(a)]. Meanwhile, the radioactivity of the organs was also measured using a gamma counter after weighing, and the radioactivity uptake in the tumor and normal tissues was calculated and expressed as a percentage of the injected radioactive dose per gram of tissue (\%ID/g) [see Fig. 3(b)]. For optical imaging of the organs, the C6-tk tumor, the kidney, and the intestine were clearly visible at 1-h postinjection. Quantification analysis also revealed that they were the organs with the highest optical signals, while all the other organs displayed minimum light intensities [see Fig. 3(a)]. It was also found that biodistribution patterns obtained through these two approaches were consistent [see Figs. 3(a) and 3(b)]. Compared with the intestines, much higher signals from the kidney are observed, which indicates that the clearance route of $\left[{ }^{18} \mathrm{~F}\right] \mathrm{FHBG}$ is mainly through urinary excretion.

The ratio of kidney to tumor uptake of $\left[{ }^{18} \mathrm{~F}\right] \mathrm{FHBG}$ at $1 \mathrm{~h}$ was also calculated to be 2.3 versus 1.4 , for gamma counting 

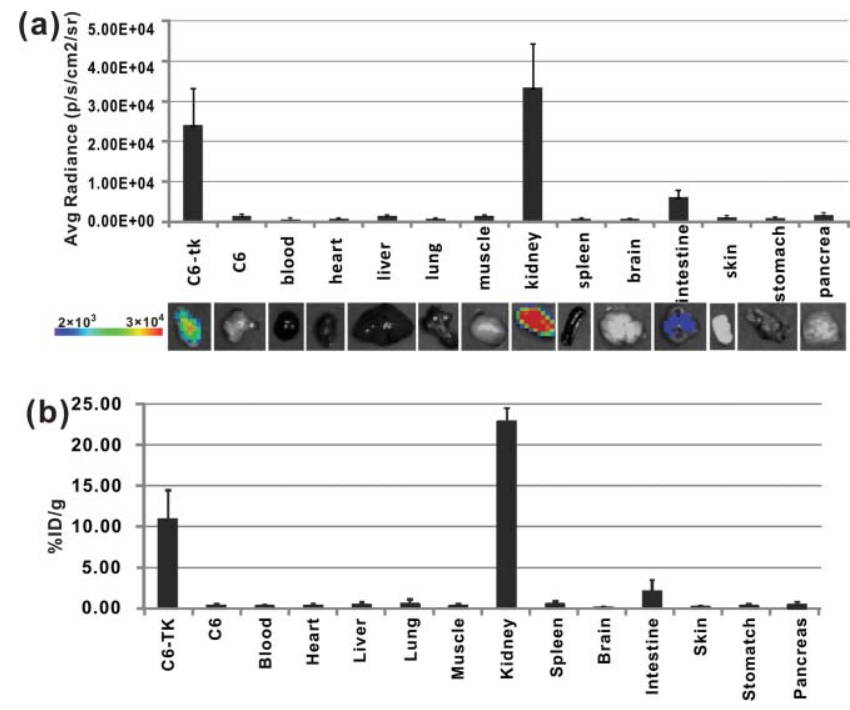

Fig. 3 Biodistribution studies measured by (a) OI or (b) gamma counting at 1 -h postinjection of $\left[{ }^{18} \mathrm{~F}\right] \mathrm{FHBG}(n=6)$.

versus OI quantification, respectively. This discrepancy could mainly originate from the diverse optical properties of different tissues, which ultimately affects light imaging and quantification. Compared with the C6-tk tumor, the kidney contains more hemoglobin that absorbs more optical photons. Moreover, unlike high energy gamma rays, the low energy photons have very weak tissue penetrability. Light sources deeper in the tissue contribute less for the total optical signals than those closer to the surface. Therefore, the kidney/tumor ratio measured by OI is lower than that obtained by gamma counting. Overall, PET has a better quantitative capability than OI. However, radioactive OI can still serve as a qualitative or semiquantitative research tool for radioactive reporter probe studies. The radioactive OI signals also have a uniquely wide wavelength of emission spectrum. ${ }^{9}$ The emissions in the near-infrared range are especially favorable for OI in living subjects. In comparison with current reporter gene imaging using BLI probes, radioactive reporter probes may offer a better option for 3-D OI reconstruction.

In conclusion, the low energy photons produced by $\left[{ }^{18} \mathrm{~F}\right] \mathrm{FHBG}$ radiation can be easily imaged by OI instruments both in vitro and in living small animals. This work for the first time demonstrates the feasibility of using OI as an alternative tool for monitoring reporter gene expression with radioactive probes. Considering the wide availability of $\mathrm{OI}$ instruments and many radioactive reporter probes such as $\left[{ }^{18} \mathrm{~F}\right] \mathrm{FHBG}$ and radioiodine, OI with radioactive reporter probes will facilitate and broaden the applications of reporter gene/reporter probe techniques in medical research.

\section{Acknowledgment}

We acknowledge support from the National Cancer Institute (NCI) R21 CA121842 (to Cheng) and a China Scholarship Council fellowship (to Liu).

\section{References}

1. J. J. Min and S. S. Gambhir, "Molecular imaging of PET reporter gene expression," Hand. Exp. Pharmacol. 185 Pt 2), 277-303 (2008).

2. T. F. Massoud and S. S. Gambhir, "Molecular imaging in living subjects: seeing fundamental biological processes in a new light," Genes Dev. 17(5), 545-580 (2003).

3. J. W. Hastings, "Chemistries and colors of bioluminescent reactions: a review," Gene 173(1 spec no), 5-11 (1996).

4. J. G. Tjuvajev, A. Joshi, J. Callegari, L. Lindsley, R. Joshi, J. Balatoni, R. Finn, S. M. Larson, M. Sadelain, and R. G. Blasberg, "A general approach to the non-invasive imaging of transgenes using cis-linked herpes simplex virus thymidine kinase," Neoplasia 1(4), 315-320 (1999).

5. S. S. Gambhir, J. R. Barrio, M. E. Phelps, M. Iyer, M. Namavari, N. Satyamurthy, L. Wu, L. A. Green, E. Bauer, D. C. MacLaren, K. Nguyen, A. J. Berk, S. R. Cherry, and H. R. Herschman, "Imaging adenoviral-directed reporter gene expression in living animals with positron emission tomography," Proc. Natl. Acad. Sci. USA 96(5), 2333-2338 (1999).

6. T. Groot-Wassink, E. O. Aboagye, M. Glaser, N. R. Lemoine, and G. Vassaux, "Adenovirus biodistribution and noninvasive imaging of gene expression in vivo by positron emission tomography using human sodium/iodide symporter as reporter gene," Hum. Gene Ther 13(14), 1723-1735 (2002).

7. A. Merron, I. Peerlinck, P. Martin-Duque, J. Burnet, M. Quintanilla, S. Mather, M. Hingorani, K. Harrington, R. Iggo, and G. Vassaux, "SPECT/CT imaging of oncolytic adenovirus propagation in tumours in vivo using the Na/I symporter as a reporter gene," Gene Ther. 14(24), 1731-1738 (2007).

8. R. Robertson, M. S. Germanos, C. Li, G. S. Mitchell, S. R. Cherry, and M. D. Silva, "Optical imaging of Cerenkov light generation from positron-emitting radiotracers," Phys. Med. Biol. 54(16), N355-365 (2009).

9. H. Liu, G. Ren, Z. Miao, X. Zhang, X. Tang, P. Han, S. S. Gambhir, and Z. Cheng, "Molecular optical imaging with radioactive probes," PLOS ONE 5(3), e9470 (2010).

10. A. E. Spinelli, D. D'Ambrosio, L. Calderan, M. Marengo, A. Sbarbati, and F. Boschi, "Cerenkov radiation allows in vivo optical imaging of positron emitting radiotracers," Phys. Med. Biol. 55(2), 483-495 (2010).

11. A. Ruggiero, J. P. Holland, J. S. Lewis, and J. Grimm, "Cerenkov luminescence imaging of medical isotopes," J. Nucl. Med. 51(7), 1123-1130 (2010)

12. S. S. Gambhir, E. Bauer, M. E. Black, Q. Liang, M. S. Kokoris, J. R. Barrio, M. Iyer, M. Namavari, M. E. Phelps, and H. R. Herschman, "A mutant herpes simplex virus type 1 thymidine kinase reporter gene shows improved sensitivity for imaging reporter gene expression with positron emission tomography," Proc. Natl. Acad. Sci. U S A 97(6), 2785-2790 (2000).

13. Z. Cheng, O. P. De Jesus, M. Namavari, A. De, J. Levi, J. M. Webster, R. Zhang, B. Lee, F. A. Syud, and S. S. Gambhir, "Small-animal PET imaging of human epidermal growth factor receptor type 2 expression with site-specific $18 \mathrm{~F}$-labeled protein scaffold molecules," J. Nucl. Med. 49(5), 804-813 (2008). 